\title{
Familial Robertsonian Translocation 13;21 in a Down Syndrome Patient with XYY/XY Mosaicism
}

\author{
Cyril Cyrus ${ }^{1}$, Teena K. ${ }^{2}$, Solomon F.D.Paul ${ }^{2}$, Chandra N. ${ }^{1}$, Meena J. ${ }^{3}$, Anuradha D. ${ }^{3}$, \\ Ramesh A. ${ }^{1}$, Gopinath P.M. ${ }^{4}$ and Marimuthu K.M. ${ }^{5}$ \\ ${ }^{1}$ Department of Genetics, Dr. ALM. PG. Institute of Basic Medical Sciences, University of \\ Madras, Taramani, Chennai 600113 \\ ${ }^{2}$ Department of Human Genetics, Sri Ramachandra Medical College \& Research Institute \\ (Deemed University), Porur, Chennai 600116 \\ ${ }^{3}$ Department of Medical Genetics, Institute of Obstetrics and Gynecology, Madras Medical \\ College, Government Hospital for Women and Children, Egmore, Chennai 600008 \\ ${ }^{4}$ KMC Life Sciences Center, Manipal Academy of Higher Education, Manipal-576104 \\ ${ }^{5}$ 26, I Main Road, Indira Nagar, Adyar, Chennai 600020
}

KEYWORDS Down syndrome; mosaicism; Robertsonian translocation; XYY males

\begin{abstract}
Double aneuploidy involving XYY and trisomy 21 is rare. XYY/XY mosaicism has been described in only a single Down syndrome patient. The Robertsonian translocation $\mathrm{t}(13 ; 21)$ is also rare among these individuals. We report for the first time the occurrence of $\mathrm{t}(13 ; 21)$ in a mosaic XYY Down male. Analysis of GTG-banded metaphases revealed the karyotype of the propositus to be mos $47, \mathrm{XYY}, \operatorname{der}(13 ; 21)(\mathrm{q} 10 ; \mathrm{q} 10),+21 /$ $46, X Y, \operatorname{der}(13 ; 21),+21$. Both his father and paternal grandfather were found to be carriers for the translocation. This 10-month-old child who presented with typical features of Down syndrome, developed leukemia and died at the age of $2^{1 / 4}$ years.
\end{abstract}

\section{INTRODUCTION}

Aneuploidy is the most frequently observed chromosomal abnormality in human liveborns, abortuses, blastomeres and oocytes. It is reported to occur in at least 3-4 percent of all clinically recognized pregnancies. XYY is a common sex chromosomal aneuploidy with an estimated incidence of 1 in 1000 male live births (Nussbaum et al. 2001). However, these cases usually go undetected during childhood due to inconsistent phenotypic features. Down syndrome (DS) or trisomy 21, with its characteristic clinical features is the most common autosomal aneuploidy with an incidence of 1:700. Double aneuploidies involving chromosome 21 and sex chromosomes have been occasionally reported. Parmar et al. (2002) found 17 published cases of 48,XYY,+21 in Medline 1965-2002 while describing an infant having the same karyotype. There was only one case of XYY Down syndrome male showing mosaicism for the $\mathrm{Y}$ chromosome (Schwanitz and

Address for all correspondence: Dr. N. Chandra, Department of Genetics, Dr. ALM. PG. Institute of Basic Medical Sciences, University of Madras, Taramani, Chennai 600 113, Tamil Nadu, India

Telephone: 044-24480769, Fax: 044-24926709

E-mail: chandrarsn@yahoo.co.in
Hagner 1978). The clinical features of DS were apparent in these individuals. XYY males are generally fertile and bear normal children while males with DS are sterile. Thus, it would be reasonable to propose that DS males with XYY chromosome pattern may also be infertile (Parmar et al. 2002).

Robertsonian translocations are known to account for 4-5 percent of all DS cases. These translocations are common balanced chromosomal rearrangements with an estimated incidence of 1 in 1000 in the general population. They can occur de novo with a high mutation rate of $3.92 \times 10^{-4}$ per gamete per generation (Jacobs 1981; Vogel and Motulsky 1997; Berend et al. 1998). The carriers of these translocations are usually phenotypically normal. This paper describes a child with Down syndrome who was found to be an XYY/XY mosaic and carried a Robertsonian translocation $\mathrm{t}(13 ; 21)$ transmitted down from his paternal grandfather. This is the first report of an XYY Down syndrome individual with $\mathrm{t}(13 ; 21)$.

\section{MATERIALS AND METHODS}

PHA-stimulated lymphocyte cultures were set up and metaphase preparations were obtained 
following the method of Hungerford (1965) from the patient, his parents and unaffected sister. Fifty GTG-banded (Seabright 1971) metaphases were analyzed from each individual. His grandparents and paternal uncle were also investigated. Chromosomal anomalies were designated as per the guidelines laid down by the nomenclature committee (ISCN 1995). Metaphases were photographed under oil immersion lens using NOVA film in Nikon photomicroscope (Labophot 2) for karyotype preparation.

\section{CASE REPORT}

A 10-month-old male, second-born of a 24year-old mother and 35-year-old father, was referred for chromosomal analysis because of dysmorphic features suggestive of Down syndrome. The infant was delivered at term following an uneventful gestation. The proband cried at birth and weighed $2.75 \mathrm{~kg}$. The proband exhibited delayed developmental milestones. The typical features observed were open fontanelle, brachycephaly, slanting palpebral fissures, epicanthal folds, low set ears, flat nasal root, open mouth, short broad hands, simian crease on the left hand, clinodactyly, hypotonia, gap between $1^{\text {st }}$ and $2^{\text {nd }}$ toes and plantar furrow. The child was reported to have suffered from frequent infections. On follow-up, he was found to have developed acute myeloid leukemia at the age of
$2 \frac{1}{4}$ years and died. The couple also has a healthy 31/2-year-old daughter.

\section{RESULTS}

The patient was found to have Down syndrome with an inherited Robertsonian translocation and also, XYY/XY mosaicism - mos 47, XYY,der(13;21)(q10;q10),+21 [64]/ 46,XY,der(13;21),+21[36] (Figs. 1 \& 2). Fifty additional metaphases were analyzed to determine the frequencies of the two cell lines. Both his father and his paternal grandfather were found to be carriers for this translocation (Fig. 3 ). The chromosomal constitution of his mother and elder sister was normal. His paternal grandmother and uncle also showed a normal karyotype. However, his paternal aunt and her two children were not available for study (Fig. 4).

\section{DISCUSSION}

Cytogenetic surveys of neonates have revealed that approximately one in 500 males is born with an extra sex chromosome (Abramsky and Chapple 1997). The commonest indication for a 47,XYY male to be karyotyped was reported to be developmental delay and / or behaviour problems. However, most males having an extra Y chromosome will go through life without a cytogenetic investigation due to inconsistent phenotypic features. The occurrence of XYY and

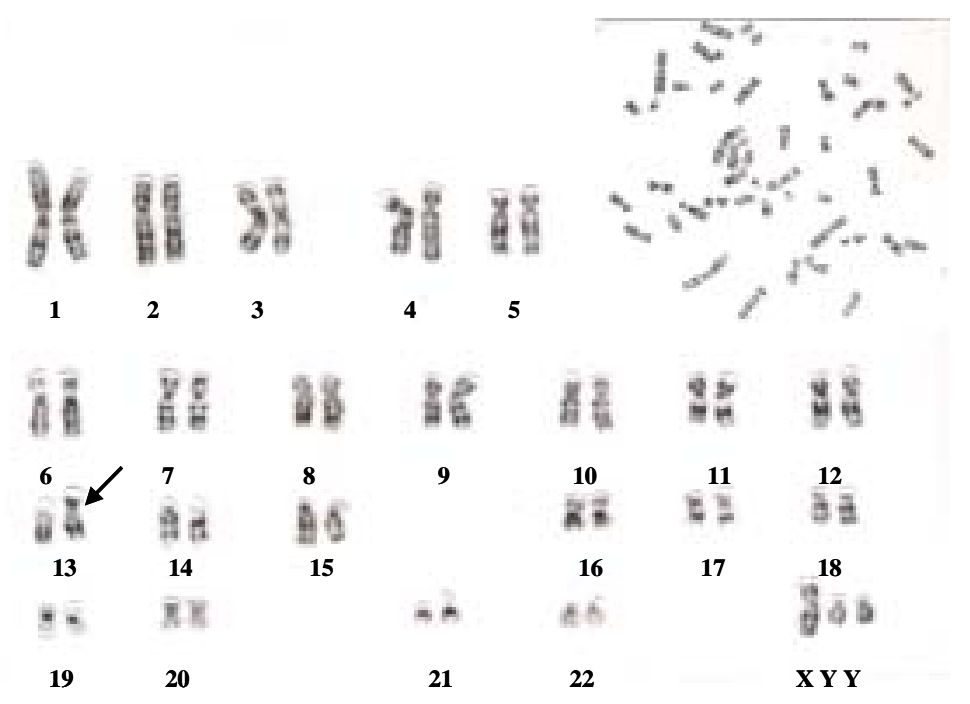

Fig. 1. A GTG - banded karyotype of the proband exhibiting 47,XYY,der(13;21)(q10;q10),+21 


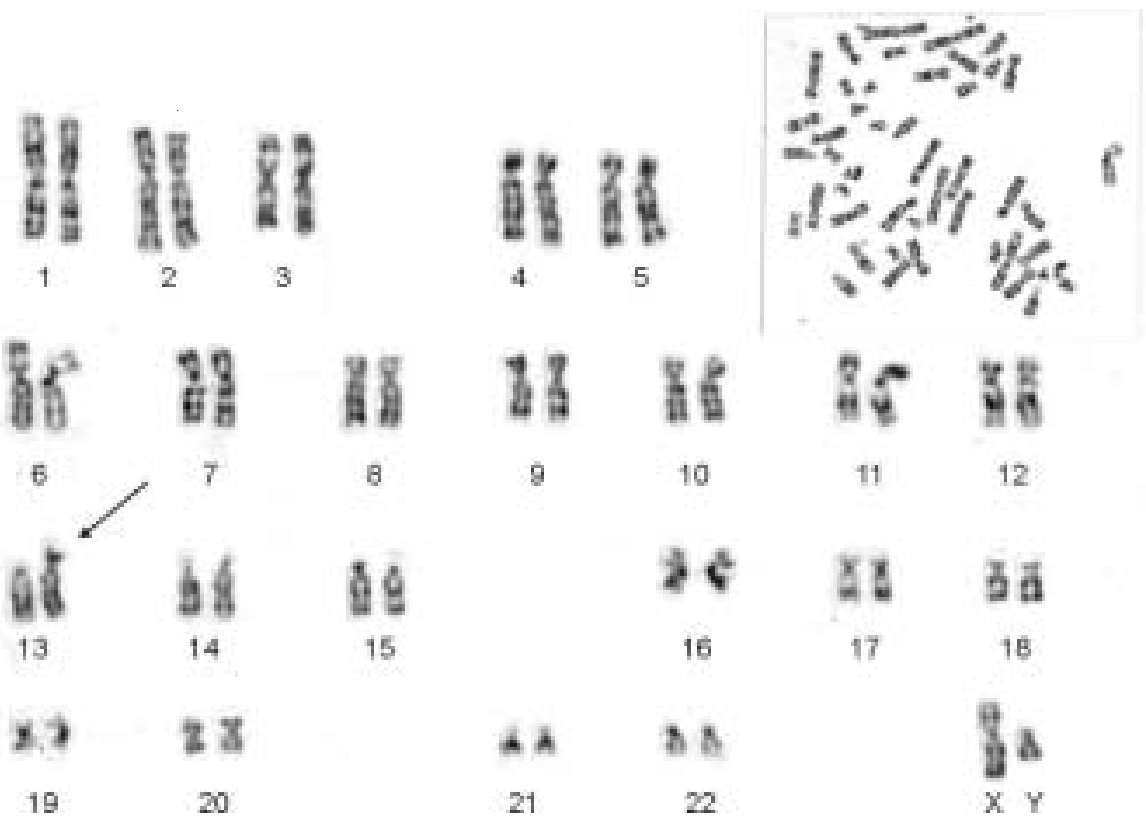

Fig. 2. A GTG - banded karyotype of the proband exhibiting 46,XY,der(13;21)(q10;q10),+21

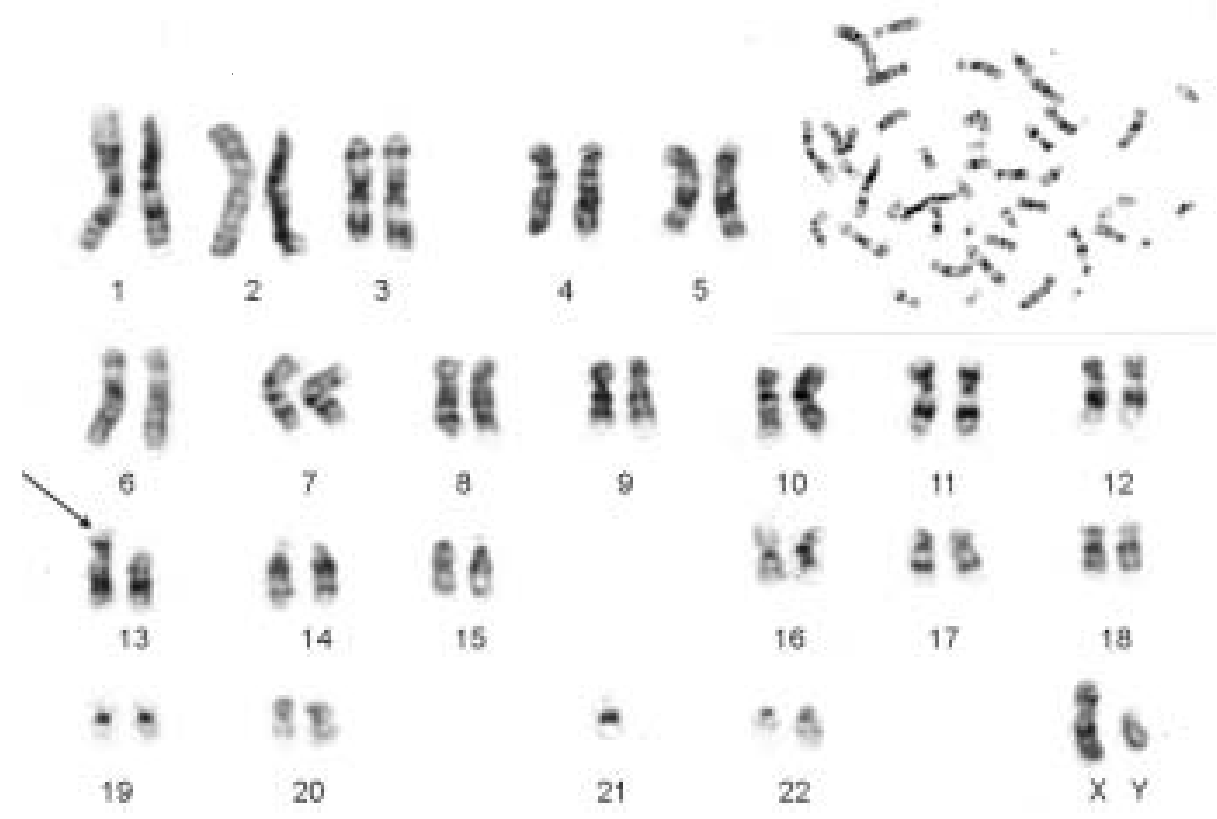

Fig. 3. A GTG - banded karyotype of the proband's father showing $45, X Y, \operatorname{der}(13 ; 21)(q 10 ; q 10)$ 

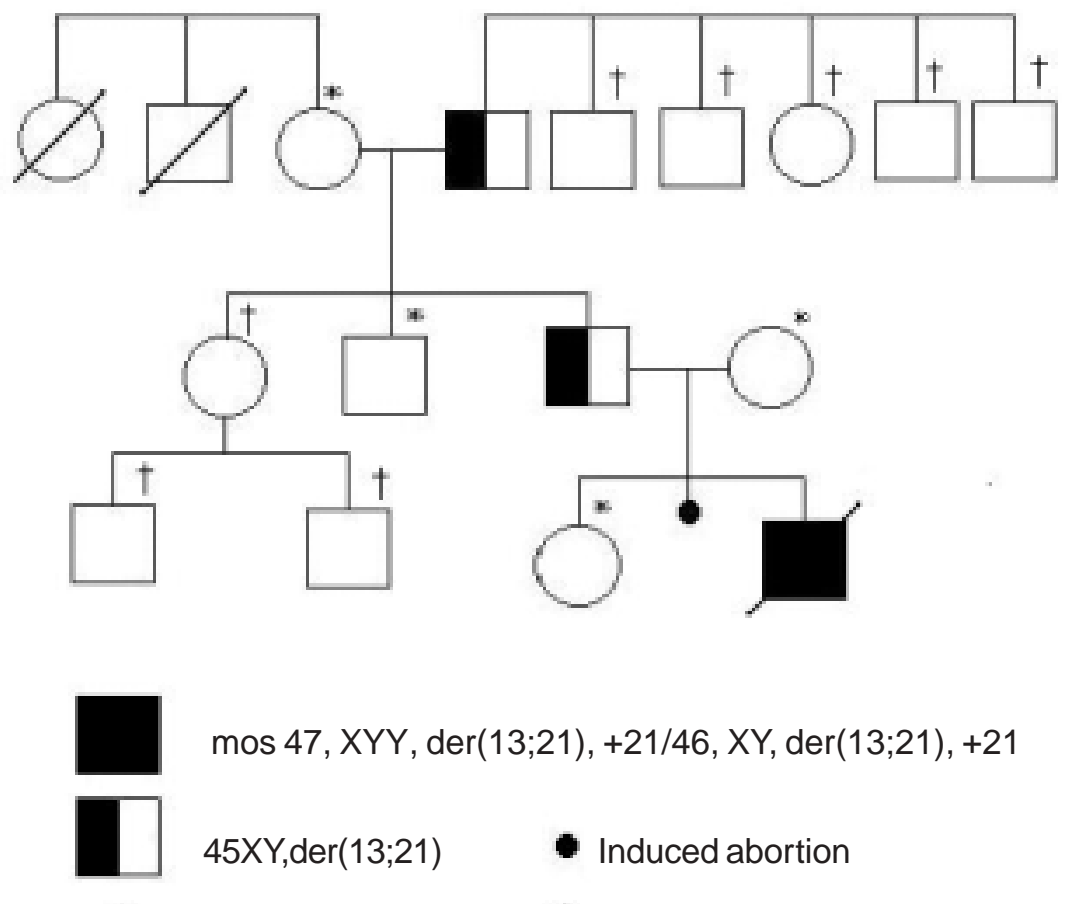

mos 47, XYY, $\operatorname{der}(13 ; 21),+21 / 46, \mathrm{XY}, \operatorname{der}(13 ; 21),+21$

$45 X Y, \operatorname{der}(13 ; 21)$

Induced abortion

* Normal karyotype

$\uparrow$ Not tested

Fig. 4. Pedigree

trisomy 21 in the same individual is a rare event. Clinical manifestations of Down syndrome remain unmasked suggesting that the presence of an additional $\mathrm{Y}$ chromosome does not produce any significant phenotypic variation (Al-Aish et al. 1971). Leary et al. (1975) could not recognize any feature that distinguished their patient from others with Down syndrome even after following their case for 5 years.

The extra Y chromosome is always paternal in origin and results from nondisjunction at the second meiotic division. Mosaicism seen in our patient may have arisen from post-zygotic mitotic nondisjunction (Jacobs and Hassold 1995). The propositus also showed a paternally inherited Robertsonian translocation $\operatorname{der}(13 ; 21)(\mathrm{q} 10 ; \mathrm{q} 10)$. Although all combinations of acrocentric chromosomes have been reported, the two translocations $\mathrm{t}(13 \mathrm{q} 14 \mathrm{q})$ and $\mathrm{t}(14 \mathrm{q} 21 \mathrm{q})$ are most common (Nussbaum et al. 2001). The extra chromosome 21 could have been of maternal origin with nondisjunction occurring at either meiosis I or II. However, it could also have been of paternal origin resulting from adjacent-I segregation at meiosis I. Carriers of Robertsonian translocations involving chromosome 21 are usually phenotypically normal but are at risk of producing unbalanced gametes and therefore producing a child with translocation Down syndrome (Nussbaum et al. 2001).

This case has been reported for its rarity and unique combination of aneuploidy, translocation and mosaicism.

\section{REFERENCES}

Abramsky L, Chapple J 1997. 47,XXY (Klinefelter syndrome) and 47,XYY: Estimated rates of and indication for postnatal diagnosis with implications for prenatal counseling. Prenat Diagn, 17: 363368 .

Al-Aish MS, Dodson WE, Plato CC 1971. Down's syndrome with XYY: 48,XYY, G+. Amer J Dis Child, 121: 444-446.

Berend SA, Canun S, McCaskill C, Page SL, Shaffer LG 1998. Molecular analysis of mosaicism for two different de novo acrocentric rearrangements 
demonstrates diversity in Robertsonian translocation formation. Am J Hum Genet, 80: 252259

Hungerford A 1965. Leucocytes cultured from small inocula of whole blood and the preparation of metaphase chromosomes by treatment with hypotonic KCl. Stain Technology, 40: 333-338

Jacobs PA 1981. Mutation rates of structural chromosome rearrangements in man. Am J Hum Genet, 33: 44-54

Jacobs PA, Hassold TJ 1995. The origin of numerical chromosome abnormalities. Adv Genet, 33: 10133.

Leary PM, Webb PM, Melzer CW, Close HG 1975. Down's syndrome with additional XYY aneuploidy. Clin Genet, 8: 55-58.

Mitelman F 1995. An International System for Human
Cytogenetic Nomenclature. Basel: S.Karger.

Nussbaum RL, McInnes RR, Willard HF 2001. Thompson

\& Thompson Genetics in Medicine. $6^{\text {th }}$ Edition, Philadelphia: W.B.Saunders Company, pp.149

Parmar RC, Muranjan MN, Swami S 2002. Trisomy 21 with XYY. Ind J Pediatr, 69: 979-981

Schwanitz G, Hagner M 1978. Double trisomy as a mosaic - Case history (48, XYY,+21/47,XY,+21) and survey of the literature of mixed autosomalgonosomal trisomies. Acta Genet Med Gemellol (Roma), 27: 67-74

Seabright M 1971. A rapid banding technique for human chromosomes. Lancet, ii: 971-972

Vogel F, Motulsky AG 1997. Human Genetics - Problems and Approaches. Berlin: Springer-Verlag, pp. 396397 